Abstracted/indexed in Academic Search Complete, Asia Journals Online, Bangladesh Journals Online, Biological Abstracts, BIOSIS Previews, CAB Abstracts, Current Abstracts, EMBASE/Excerpta Medica, Google Scholar, HINARI (WHO), International Pharmaceutical Abstracts, Open J-gate, Science Citation Index Expanded, SCOPUS and Social Sciences Citation Index;

ISSN: $1991-0088$

\title{
In vitro and in situ effects of atorvastatin and ezetimibe on P-glycoprotein expression and function
}

\author{
Mehran Mesgari Abbasi', Hadi Valizadeh', Hamed Hamishehkar'1, Maryam Bannazadeh \\ Amirkhiz ${ }^{1}$ and Parvin Zakeri-Milani²
}

${ }^{1}$ Drug Applied Research Center, Tabriz University of Medical Sciences, Tabriz, Iran; ${ }^{2}$ Liver and Gastrointestinal Diseases Research Center and Faculty of Pharmacy, Tabriz University of Medical Sciences, Tabriz, Iran.

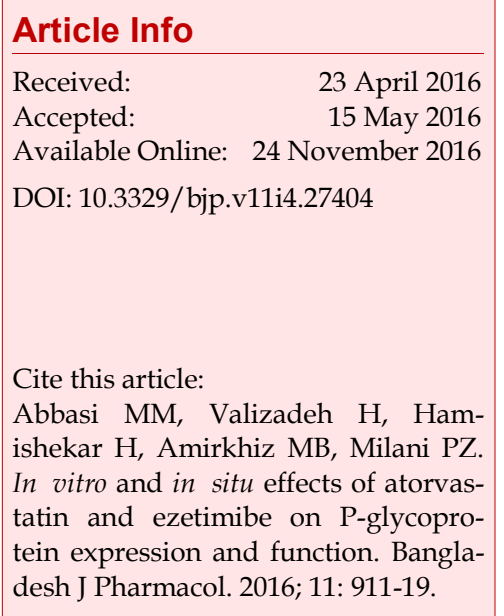

\begin{abstract}
P-glycoprotein (P-gp) is a membrane transporter responsible for the active efflux from the cell. Inhibition of the activity may lead to clinically significant drug-drug interactions. This study was performed to investigate the effects of atorvastatin and ezetimibe on the function and expression of P-gp. The in vitro rhodamine-123 (Rho123) efflux assay and Western blot in Caco-2 cells, and the in situ rat single-pass intestinal permeability model followed by high performance liquid chromatography were developed. Rho123 intracellular accumulation in $100 \mu \mathrm{M}$ of atorvastatin- and ezetimibe-treated cells was significantly higher than that in control cells $(p<0.05)$. P-gp expression was decreased by $100 \mu \mathrm{M}$ atorvastatin and ezetimibe. Intestinal effective permeability of digoxin in the presence of atorvastatin ( 3 and $100 \mu \mathrm{M})$, ezetimibe $(10$ and $100 \mu \mathrm{M})$ was significantly increased $(\mathrm{p}<0.05)$. Both drugs inhibited P-gp activity in vitro and in situ. Atorvastatin and ezetimibe downregulated the expression of $\mathrm{P}$-gp in vitro.
\end{abstract}

\section{Introduction}

In recent years, the potential for significant clinical drug -drug interactions (DDIs) has emerged owing to the growing use of drugs and herbal medicines. Most drugs are administered orally and must, therefore, be adequately and consistently absorbed to achieve successful therapy. Membrane transporter is an important risk factor for developing DDI. The active efflux of drugs from enterocytes back into the intestinal lumen is one of the major barriers to oral drug delivery.

P-glycoprotein (P-gp), an ATP binding cassette protein, is a membrane transporter responsible for the active efflux and the secretion of passively diffused drugs and xenobiotics from the cell. It is expressed on several barrier epithelia, including the small intestine, bloodbrain barrier, liver, kidneys, and bile canaliculi. This multidrug transporter has broad substrate specificity, with a tendency towards lipophilic, cationic com- pounds. Thus, P-gp plays critical role in the absorption and excretion of drugs, and the distribution of drugs to various physiological spaces and target tissues.

The transport activity of P-gp can be inhibited in vivo by a variety of drugs, such as verapamil, ketoconazole, and cyclosporine, which may affect the absorption of the drugs themselves and that of concomitantly used drugs.

Competitive or non-competitive inhibition of P-gp by drugs or other xenobiotics may lead to pronounced inter-individual variability of its transport activity, resulting in clinically significant DDIs. DDIs affect the drugs' bioavailability and effectiveness and may cause drug toxicity and unwanted adverse effects. Therefore, owing to the clinical importance of P-gp related DDIs, it is critical to identify drugs that are inhibitors of P-gp so that adverse DDIs might be minimized or avoided (Ando et al., 2015; Li et al., 2014; O'Connor et al., 2015). 
Atorvastatin, an effective hypolipidemic synthetic drug, is one of the most widely prescribed drugs and the most widely prescribed statin worldwide. Atorvastatin is an inhibitor of 3-hydroxy-3-methylglutaryl-coenzyme A reductase (HMG CoA), which catalyzes the conversion of HMG CoA to mevalonate, an early rate limiting step in the cholesterol biosynthesis pathway. By inhibiting this enzyme, atorvastatin reduces hepatocyte cholesterol levels, resulting in up-regulation of lowdensity lipoprotein (LDL) receptors and, consequently, increased clearance of LDL-cholesterol (LDL-C) from the blood plasma. It reduces total cholesterol, LDL, and triglyceride (TG) while elevating the blood level of high -density lipoprotein (HDL). It shows poor oral bioavaila -bility (12\% in humans and $6 \%$ in rats), poor solubility, and extensive hepatic first-pass biotransformation, preventing its effectiveness in most chemotherapeutic applications (Adams et al., 2012; Horwich and McLellan, 2007; Rodde et al., 2014; Vatsa et al., 2012).

Ezetimibe or 1-(4-fluorophenyl)-(3R)-[3-\{4-fluorophenyl\}-\{3S\}-hydroxyprophyl]-(4S)-(4-hydroxyphenyl)-(2azetidinone) is widely used as a lipid lowering agent and, unlike statins, inhibits intestinal and biliary cholesterol and phyto-sterol absorption by selectively blocking the Niemann-Pick C 1-like 1 protein (NPC1L1) in the brush border of jejunum and, to a lesser extent, in the duodenum and the ileum. It can significantly lower LDL-C and non-high-density lipoprotein cholesterol (non-HDL-C) when used alone or in combination with statin therapy. Ezetimibe prevents the NPC1L1/sterol complex from interacting with AP2 in clathrin-coated vesicles. It may change the shape of NPC1L1 to render it incapable of binding to sterols or interfere with the binding of free cholesterol to the cell membrane. After oral administration, it is rapidly absorbed from the gut lumen and conjugated by UDP-glucuronosyl-transferases (Bandyopadhyay et al., 2012; Lioudaki et al., 2011; Oswald et al., 2006a; Oswald et al., 2006b; Oswald et al., 2007; Phan et al., 2012; Suchy et al., 2011).

The purpose of this study was to investigate the in vitro and in situ effects of atorvastatin and ezetimibe on the membrane transporter function of P-gp. The effects of the drugs on P-gp expression in Caco-2 cells were also investigated.

\section{Materials and Methods}

\section{Materials}

Anti-P-gp mouse monoclonal antibody (ab80594) and rabbit polyclonal antibody to $\beta$-actin (ab16039) were purchased from Abcam (UK). Horseradish peroxidase (HRP)-conjugated anti-mouse immunoglobulin (IgG) (AP8036) and anti-rabbit IgG (AP7181) were purchased from Razi Biotech (Iran). Rhodamine-123 (Rho123), digoxin, verapamil, atorvastatin, ezetimibe, 3-(4,5-dime- thylthiazol-2-thiazolyl)-2,5-diphenyl-tetrazoliumbromide (MTT), penicillin-streptomycin, protease inhibitor cocktail, dimethyl sulfoxide (DMSO), and non-fatdried bovine milk were purchased from Sigma-Aldrich (USA). Dulbecco's Modified Eagle Medium (DMEM), trypsin-EDTA $(0.25 \%)$, and fetal calf serum (FCS) were purchased from Gibco (USA). Tissue culture flasks and other disposable cell culture items were purchased from TPP Co. (Switzerland). Enhanced chemiluminescence (ECL) Western blotting detection kit, medical X-ray film, pre-stained protein ladder $(10-250 \mathrm{kDa})$, thiopental sodium, and protein assay kit were purchased from Amersham (UK), Fuji (Japan), Cinagen (Iran), LogiChem (UK), and Pars Azmoon (Iran), respectively. All other chemicals were purchased from Merck Co. (Germany).

Since atorvastatin and ezetimibe are only slightly soluble in water, atorvastatin and ezetimibe were primarily dissolved in methanol and ethanol, respectively, for the initial stock solution, which was diluted to produce the needed working solutions (Oswald et al., 2006a; Rodde et al., 2014). Vehicle controls were performed with methanol and ethanol (without drugs) to control for their potential effects.

\section{In vitro study}

\section{Cell culture}

The Caco-2 cells were purchased from Pasteur Institute of Iran (Iran) and were cultured in DMEM containing $10 \%$ fetal calve serum and $1 \%$ penicillin-streptomycin. The culture flasks were incubated in a $\mathrm{CO}_{2}$-incubator (Germany) at $37^{\circ} \mathrm{C}$ with a $5 \% \mathrm{CO}_{2}$ atmosphere. The culture medium was changed 2-3 times per week. After 2-3 weeks culture and differentiation of Caco-2 into intestinal-like cells, the cells were detached from the culture flask by addition of $0.25 \%$ trypsin-EDTA. The cells were then washed with phosphate buffer solution $(\mathrm{pH}=7.4)$ and seeded at the needed density for the following experiments.

\section{Cytotoxicity study (MTT assay)}

The cytotoxicity of different concentrations of atorvastatin and ezetimibe were investigated using the MTT assay. Caco- 2 cells were seeded into 96-well plates at a density of $15 \times 10^{3}$ cells per well. After 24 hours, the medium was replaced with $200 \mu \mathrm{L}$ per well of atorvastatin and ezetimibe at different concentrations (diluted with complete culture medium) or complete culture medium as control. Three wells for each concentration or control were considered. After 24 hours, the MTT solution was added to each well at final concentration of $2 \mathrm{mg} / \mathrm{mL}$ and incubated for 4 hours at $37^{\circ} \mathrm{C}$ in the $\mathrm{CO}_{2}$ incubator. The MTT solution was discarded and the produced formazan crystals were solubilized with $200 \mu \mathrm{L} /$ well of DMSO and $25 \mu \mathrm{L} /$ well Sorensen buffer. The optical densities (ODs) were 
measured with an enzyme linked immunosorbent assay (ELISA) micro-plate reader (Statfax-2100, Awareness, USA) at $570 \mathrm{~nm}$ with background subtraction at 630 $\mathrm{nm}$. The percentage of cell viability was calculated using the following formula:

Cell viability $(\%)=\frac{\text { OD of test }}{\text { OD of control }} \times 100$

The mean \pm standard deviation (SD) was calculated for $0.1,3$, and $100 \mu \mathrm{M}$ concentrations of atorvastatin and $0.2,10$, and $100 \mu \mathrm{M}$ concentrations of ezetimibe.

\section{Rho123 uptake assay}

Rho123 is a well-known P-gp substrate and is secreted by Caco-2 cells via P-gp efflux. Treatment with an inhibitor of P-gp (verapamil) will promote the accumulation of Rho123 in Caco-2 cells. In this study, Caco-2 cells were seeded in 24-well plates and allowed to attach for 24 hours. The old medium was removed, and cells were washed with PBS. Then, new culture media containing $100 \mu \mathrm{M}$ atorvastatin, $100 \mu \mathrm{M}$ ezetimibe, or $300 \mu \mathrm{M}$ verapamil without FCS or fetal bovine serum (FBS) were added to separate wells and incubated. Because of the protein binding properties of atorvastatin (>98\%) (Lennernäs, 2003) and ezetimibe (99.5-99.8\%) (Oswald et al., 2007), which may interfere with the results, we did not add FCS or FBS to the culture medium during this treatment step. After 24 hours, the old medium was discarded and cells were washed with PBS. Rho123 solution [DMEM containing $10 \mathrm{mM}$ HEPES ( $\mathrm{pH}=7.4$ ) and $5 \mu \mathrm{M}$ Rho123] was added and incubated for 3 hours at $37^{\circ} \mathrm{C}$, followed by three washes with ice-cold PBS ( $\mathrm{pH}=7.4)$. Cells were lysed in $1 \%$ Triton X-100 and centrifuged (3-18k, Sigma) at 1500 $g$ for $10 \mathrm{~min}$. The cellular accumulation of Rho123 in each sample (Rh-123 contents of supernatant) was measured quantitatively by fluorescence spectrophotometry $(\lambda$ ex $=485 \mathrm{~nm}$, $\lambda$ em $=532 \mathrm{~nm})($ FP-750, Jasco, Japan) and normalized by the protein content of each sample (supernatant), as determined by a protein assay kit (Pars Azmoon, Iran) (Mohammadzadeh et al., 2014; Zastrea et al., 2002; Zrieki et al., 2010).

\section{Western blotting analysis of P-gp expression}

Expression of P-gp in Caco-2 cells was assayed by Western blot after cells were treated with atorvastatin, ezetimibe, and verapamil. Caco- 2 cells were seeded in a 6-well plates at $10^{6}$ cells per well and treated for 48 hours with $100 \mu \mathrm{M}$ atorvastatin, $100 \mu \mathrm{M}$ ezetimibe, or $300 \mu \mathrm{M}$ verapamil in culture medium in separate wells $(\mathrm{n}=4)$. Culture medium alone was considered the control $(n=4)$. Solutions were then discarded, and cells were washed with PBS and incubated for $5 \mathrm{~min}$ with $0.25 \%$ trypsin/EDTA in $37^{\circ} \mathrm{C}$. The detached cells were washed twice with PBS. Lysis buffer (Triton X-100 50 $\mathrm{mM}, \mathrm{NaCl} 150 \mathrm{mM}$, EDTA $5 \mathrm{mM}, 1 \%$ protease inhibitor cocktail, Tris- $\mathrm{HCl}, \mathrm{pH}=7.4$ ) was added and cell suspen- sions were centrifuged at 1,500 xg for $5 \mathrm{~min}$. Proteins were resolved by $12.5 \%$ sodium dodecyl sulfatepolyacrylamide gel electrophoresis (SDS-PAGE) (80 V, $120 \mathrm{~min}$ ) and electrophoretically transferred to polyvinylidene di-fluoride (PVDF) membrane using a semidry Western blotting system (Bio-Rad, Hercules, CA, USA). All membranes were blocked in 3\% non-fat-dried milk for 1 hour at room temperature and then washed three times with Tris buffered saline (TBS) with $0.1 \%$ Tween 20). Membranes were subsequently incubated overnight with mouse monoclonal anti-P-gp antibody (1/1000 in TBS). Membranes were washed with TBS and then incubated with HRP-conjugated rabbit antimouse secondary antibody for 2 hours. Membranes were washed, and the proteins were then detected using an ECL kit. The proteins were visualized by exposing the membrane to medical X-Ray film for 5 min. $\beta$-Actin was the internal standard and was detected using rabbit polyclonal anti- $\beta$-actin as primary antibody and HRP-conjugated goat anti-rabbit IgG as secondary antibody. P-gp expression is presented as the ratio of $\mathrm{P}$-gp band intensity to $\beta$-actin band intensity in the same blot run (P-gp/ $\beta$-actin) (Wanga et al., 2009).

\section{In situ study}

The rat single-pass intestinal permeability experiment with P-gp inhibition study was used to investigate whether intestinal P-gp-mediated transport of digoxin, a known substrate of P-gp, was affected by atorvastatin and ezetimibe. The effective permeability of digoxin was calculated and compared in the presence and absence of atorvastatin, ezetimibe and verapamil.

\section{Animals}

Male Sprague-Dawley rats (200-250 g) were purchased from Pasteur Institute of Iran. The rats were housed in standard cages under standard husbandry conditions (22 $\pm 2{ }^{\circ} \mathrm{C}, 12 / 12$ hours light/dark cycle and 55-60\% relative humidity). Standard rodent chow and clean water were available ad libitum. Prior to experimentation, rats were fasted for 12-16 hours (water ad libitum).

\section{P-gp inhibition study by single-pass intestinal perfusion test (Video Clip)}

The rats were anesthetized with an intraperitoneal injection of thiopantal sodium ( $60 \mathrm{mg} / \mathrm{kg}$ body weight). A midline abdominal incision of $3-4 \mathrm{~cm}$ was made and approximately $10 \mathrm{~cm}$ of the jejunum segment of the intestine was isolated and cannulated at both ends with polypropylene tubes. Body tempered saline was used for moisturizing the exposed segment. The segment was rinsed with $37^{\circ} \mathrm{C}$ normal saline to wash and clear the segment, then PBS ( $\mathrm{pH}=7.4)$ containing $50 \mathrm{mg} / \mathrm{L}$ phenol red without drug (blank solution) was pumped through the segment at a constant flow rate of $0.2 \mathrm{~mL} /$ min $\left(Q_{\text {in }}\right)$. Blank perfused solution was collected at the outlet and used to prepare digoxin high performance liquid chromatography (HPLC) calibrator solutions and 
for stability studies (Patel and Barve, 2012; ZakeriMilani et al., 2007).

The SPIP method was carried out in anesthetized rats as described above, and the blank solution containing the substance of interest was perfused at a constant flow rate of $0.2 \mathrm{~mL} / \mathrm{min}$ using a volumetric infusion pump (Argus Medical AG, Switzerland). After reaching the steady state, each perfusion experiment lasted for 90 min, and perfusates were quantitatively collected at 10 min intervals $(2 \mathrm{~mL})$. Some changes, such as water absorption and secretion, during perfusion may cause errors in the calculated permeability values. Therefore, $50 \mathrm{mg} / \mathrm{L}$ phenol red was added as a non-absorbable marker to correct the results. Digoxin $(20 \mu \mathrm{M}$, a typical substrate of P-gp), verapamil (300 $\mu \mathrm{M}$, a typical P-gp inhibitor), and different concentrations of atorvastatin $(0.1,3$ and $100 \mu \mathrm{M})$ and ezetimibe $(0.2,10$ and $100 \mu \mathrm{M})$, each one in a blank solution with digoxin $20 \mu \mathrm{M}$, were administrated, as described above (Patel and Barve, 2012; Zakeri-Milani et al., 2007). The SPIP method was performed in three rats for each concentration of each drug and the blank solution. At the end of procedure, the length of the segment was measured $(\mathrm{cm})$ and the animal was euthanatized. Samples were stored in an ultra-low temperature freezer (Jal Tajhiz Production, Iran) at $-70^{\circ} \mathrm{C}$ until HPLC analysis. The concentrations of phenol red in the perfused (outlet) samples were measured at $560 \mathrm{~nm}$ using an ultraviolet-visible (UVVIS) spectrophotometer (Ultra-spec 2000, Pharmacia, Pfizer, USA) (Valizadeh et al., 2012). The amount of digoxin in the perfused samples was detected by HPLC.

\section{HPLC analysis of digoxin in perfused samples}

HPLC system (Smartline manager 5000, Smartline UV detector 2600 and Smartline pump 1000, Knauer Advanced Scientific Instruments, Germany) was used to detect the amount of digoxin in the perfused samples. The mobile phase was $35 \% \mathrm{v} / \mathrm{v}$ of acetonitrile in water, which was filtered through a sintered glass filter P5 (1.0-1.6 $\mu \mathrm{m}$, Winteg, Germany) and degassed in a sonicator. The mobile phase was pumped in isocratic mode at a flow rate of $2 \mathrm{~mL} / \mathrm{min}$ at ambient temperature. UV detection was performed at $218 \mathrm{~nm}$, and the injection volume of samples was $20 \mu \mathrm{L}$. The HPLC column was Knauer-15VE081ESJ-150X $4.6 \mathrm{~mm}$ with precolumn-Eurospher 100-5 C8 (Wanga et al., 2009).

\section{Calculation and analysis of data}

Effective permeability $\left(\mathrm{P}_{\text {eff }}\right)$ values were calculated from the steady state concentrations of digoxin in the perfusate samples. Steady state was confirmed by the ratio of the outlet to inlet concentrations (corrected for water transport) versus time (Patel and Barve, 2012;

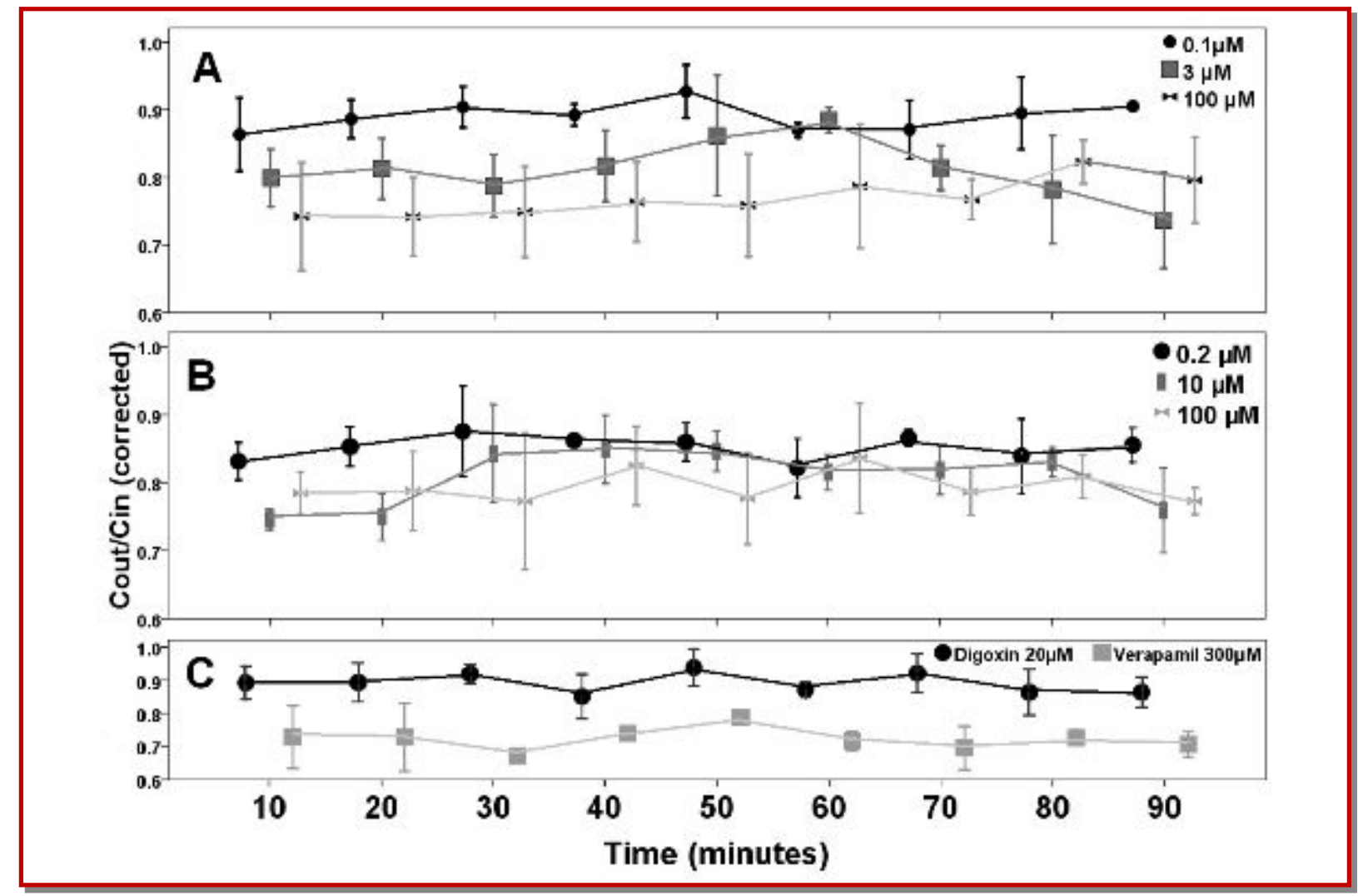

Figure 1: Plots of outlet and inlet concentrations ratio (Cout/Cin) of digoxin $(20 \mu \mathrm{M})$ vs time for atorvastatin (A), ezetimibe (B), digoxin, and verapamil $(C)$ in rat SPIP study $(n=3$, error bars represent mean $\pm S D)$ 
Zakeri-Milani et al., 2007). Figure 1 illustrates the steady state charts.

\section{Phenol red correction}

Corrected $\mathrm{C}_{\text {out }}$ (outlet concentration of the drug) was calculated from the following equation (Prasad and Bhasker, 2012).

$$
C_{\text {out (corr) }}=C_{\text {out }} \times \frac{\text { inlet phenol red conc. }}{\text { outlet phenol red conc. }}
$$

$\mathrm{C}_{\text {out }}($ corr $)=$ Corrected outlet concentration of the drug; $\mathrm{C}_{\text {out }}=$ Outlet concentration of the drug

Calculations were based on outlet perfusate steady state concentrations achieved after the selected time points (Figure 1). The steady state intestinal effective permeability $\left(\mathrm{P}_{\text {eff }}\right)$ was calculated according to following equation:

$$
P_{\text {eff }}=\frac{-Q_{\text {in }} \times \ln \left(C_{\text {out }} / C_{\text {in }}\right)}{2 \times 60 \pi r l}
$$

$P_{\text {eff }}$ represents effective permeability $(\mathrm{cm} / \mathrm{s}), Q_{\text {in }}$ represents the perfusion rate $(0.2 \mathrm{~mL} / \mathrm{min}), C_{i n}$ and $C_{\text {out }}$ represent the concentrations of the test drug entering and leaving the segment, respectively, $\mathrm{r}$ is the radius of the intestinal segment $(0.18 \mathrm{~cm})$, and $\mathrm{L}$ is the length of the intestinal segment $(\mathrm{cm})$

\section{Statistical analysis}

Data are presented as the mean \pm SD. Statistical analyses were performed using one-way analysis of variance (ANOVA) followed by Tukey's multiple comparison test, and the differences between two groups were determined using the unpaired t-test. Statistical tests were performed with SPSS13.0 (SPSS Inc., Chicago, IL, USA), where $p<0.05$ and $p<0.01$ were considered to be statistically significant.

\section{Results}

\section{In vitro study}

Cytotoxicity

The MTT cytotoxicity assay on Caco-2 cells treated with $0.1,3$, and $100 \mu \mathrm{M}$ atorvastatin, $0.2,10$, and $100 \mu \mathrm{M}$ ezetimibe, $300 \mu \mathrm{M}$ verapamil and $20 \mu \mathrm{M}$ digoxin solutions showed no cytotoxicity (Figure 2). Statistical analyses showed that there was no significant difference in cell viability among the different treatment groups, and the concentrations used were considered for the following in vitro and in situ tests.

\section{Rho123 accumulation in Caco-2 cells}

To investigate the effects of atorvastatin and ezetimibe on the function of P-gp as an efflux pump in vitro, we measured the accumulation of Rho123 in Caco-2 cells treated with atorvastatin, ezetimibe and verapamil. As shown in Figure 3, treatment with $100 \mu \mathrm{M}$ atorvastatin and $100 \mu \mathrm{M}$ ezetimibe significantly increased the intracellular accumulation of Rho123 in Caco-2 cells relative to control $(p<0.05)$. The mean intracellular concentration of Rho123 in control cells was $424.4 \pm$ 89.3, while in atorvastatin, ezetimibe and verapamil treated cells, it was $646.0 \pm 13.1,681.7 \pm 135.0$, and 749.4 $\pm 32.1 \mathrm{pg} / \mathrm{mg}$ protein respectively.

\section{Immunoblotting}

After treating Caco-2 cells with atorvastatin, ezetimibe $(100 \mu \mathrm{M})$, or verapamil $(300 \mu \mathrm{M})$, immunoblotting of cell extracts was performed using a monoclonal antibody against P-gp to measure P-gp expression. Protein expression of $\beta$-actin was measured as an internal immunoblotting control. P-gp expression is presented as the ratio of $\mathrm{P}$-gp band intensity to $\beta$-actin band intensity (P-gp/ $\beta$-actin) and was compared with verapamil control bands in the same blot run (Mohammadzadeh et al., 2014). Low density of immunoblotting bands of atorvastatin and ezetimibe treatment decreased P-gp protein levels relative to control (Figure 4).

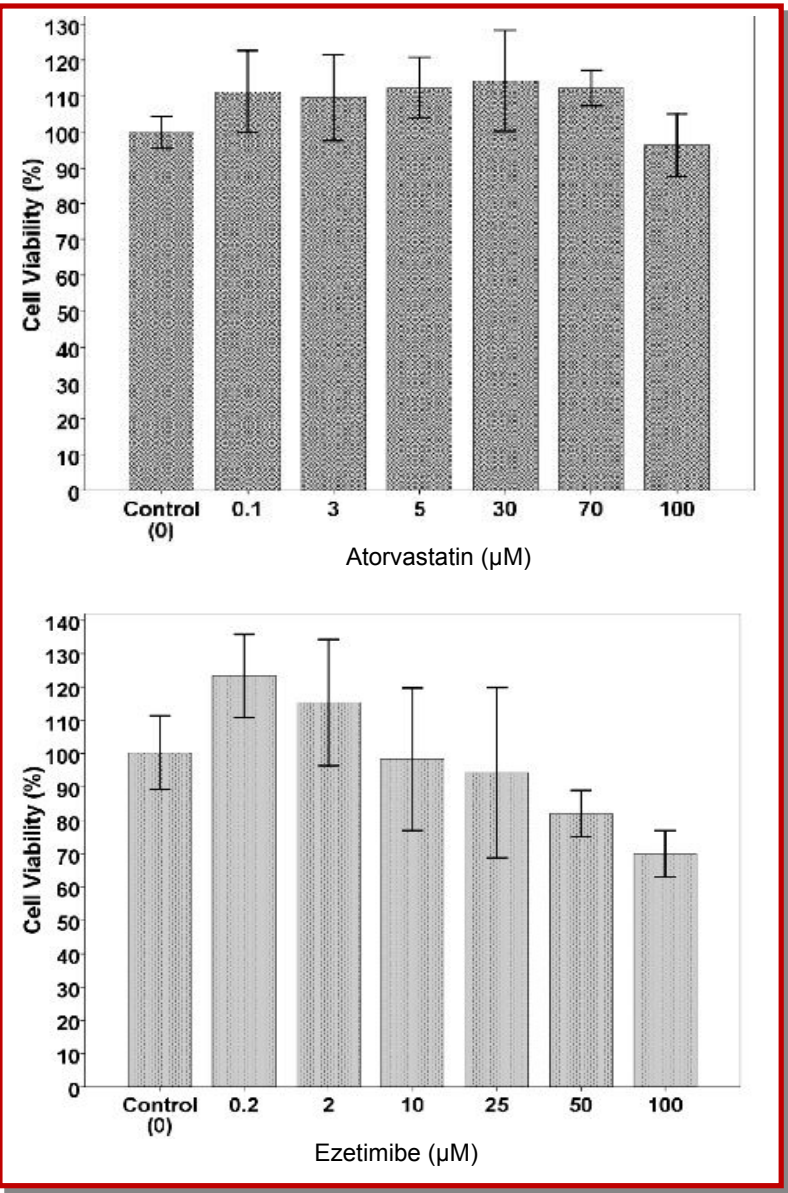

Figure 2: Effects of different concentrations of atorvastatin (upper figure) and ezetimibe (lower figure) on Caco-2 cell viability measured by MTT assay. Data are mean \pm SD of three separate experiments. The treatments were not significantly different from the untreated control at $(\mathrm{p}>0.05)$ 


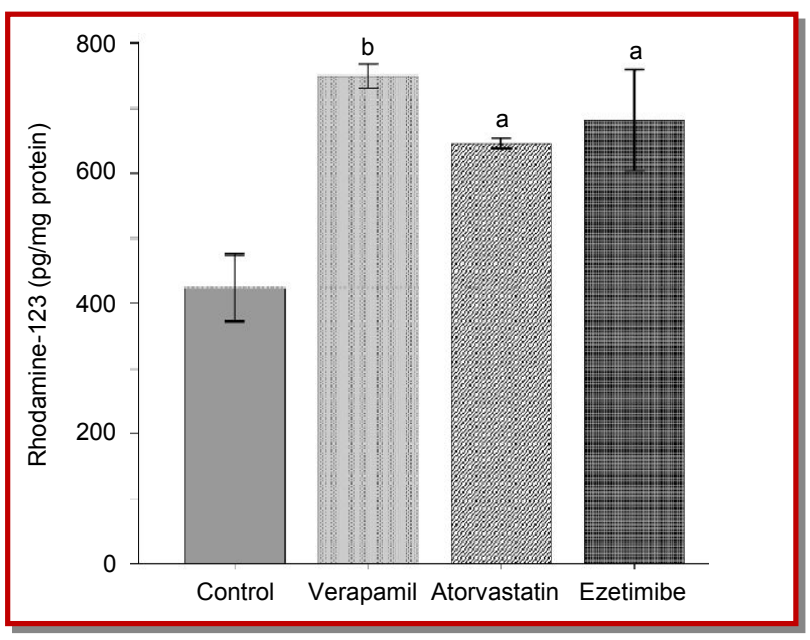

Figure 3: Effects of verapamil $(300 \mu \mathrm{M})$, atorvastatin $(100 \mu \mathrm{M})$ and ezetimibe $(100 \mu \mathrm{M})$ on intracellular accumulation of Rho123 in Caco-2 cells $(n=4)$. Bars show mean \pm SD. a and brepresent significantly different from control (untreated) cells $(\mathrm{p}<0.05$ and $\mathrm{p}<0.01$, respectively)

\section{In situ study}

\section{Adsorption experiments}

Preliminary adsorption experiments showed that there was no considerable adsorption of compounds on the tubing or syringe and that the stock and working standard solutions of digoxin and phenol red were stable during the experiments.

\section{Single-pass intestinal permeability}

The effects of the drugs on P-gp function were investigated in situ using the rat SPIP method. Inhibition of P-gp activity with atorvastatin, ezetimibe, and verapamil treatment was investigated using digoxin as the typical P-gp substrate. The $P_{\text {eff }}$ values of digoxin $(20 \mu \mathrm{M})$ in the absence and presence of verapamil were $3.4 \pm 0.8$ and $8.9 \pm 0.7 \times 10^{-5} \mathrm{~cm} / \mathrm{s}$, respectively, whereas they were $4.4 \pm 0.6,6.0 \pm 1.1$, and $7.3 \pm 0.7 \times 10^{-5} \mathrm{~cm} / \mathrm{s}$ with $0.1,3$ and $100 \mu \mathrm{M}$ atorvastatin, respectively. The $P_{\text {eff }}$ values of digoxin in the presence of $0.2,10$ and $100 \mu \mathrm{M}$ ezetimibe were $4.8 \pm 0.4,6.5 \pm 0.4$, and $6.5 \pm 1.0 \times 10^{-5} \mathrm{~cm} / \mathrm{s}$, respectively. As shown in Figure 5, $0.1 \mu \mathrm{M}$ atorvastatin did not significantly increase the $\mathrm{P}_{\text {eff }}$ of digoxin compared with the control group (digoxin alone) $(\mathrm{p}>0.05)$, but $\mathrm{P}_{\text {eff }}$ at high concentrations, 10 and $100 \mu \mathrm{M}$, was significantly different $(\mathrm{p}<0.05$ and $\mathrm{p}<0.01$, respectively). Ezetimibe $(0.2 \mu \mathrm{M})$ treatment did not significantly increase the $\mathrm{P}_{\text {eff }}$ of digoxin relative to the control group $(p>0.05)$, whereas 10 and $100 \mu \mathrm{M}$ did significantly increase $P_{\text {eff }}$ relative to control $(\mathrm{p}<0.05)$.

\section{Discussion}

Based on data from the in vitro Rho123 uptake study, treatment of Caco- 2 cells with atorvastatin increased the accumulation of Rho123 compared with control, suggesting that atorvastatin inhibits P-gp in vitro. We also found in situ that atorvastatin significantly

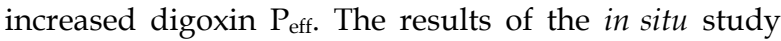
showed a dose-dependent effect of atorvastatin, in the range of $0.1-100 \mu \mathrm{M}$, on P-gp. Protein expression of Pgp in atorvastatin-treated Caco- 2 cells was low relative to control cells, confirming inhibition of P-gp by atorvastatin.

Although there are a few studies about the effect of atorvastatin on P-gp function, we did not find any evidence that is in contrast to our results. Vatsa et al. reported that atorvastatin is a moderate substrate for CYP $\mathrm{P}_{450}$ and is extensively metabolized via CYP $\mathrm{P}_{450-}$ mediated oxidation and phase II glucuronidation both in humans and rats (Vatsa et al., 2012). The drug is a CYP3A4 substrate, and most of CYP3A4 substrates are P-gp substrates as well (Boyd et al., 2000). Vatsa et al. stated that atorvastatin is a P-gp substrate at lower dose and an inhibitor at higher doses (Vatsa et al., 2012). The P-gp substrate activity of atorvastatin was also suggested by some other studies (Williams and Feely, 2002; Wu et al., 2000). Boyd et al. found that $100 \mu \mathrm{M}$

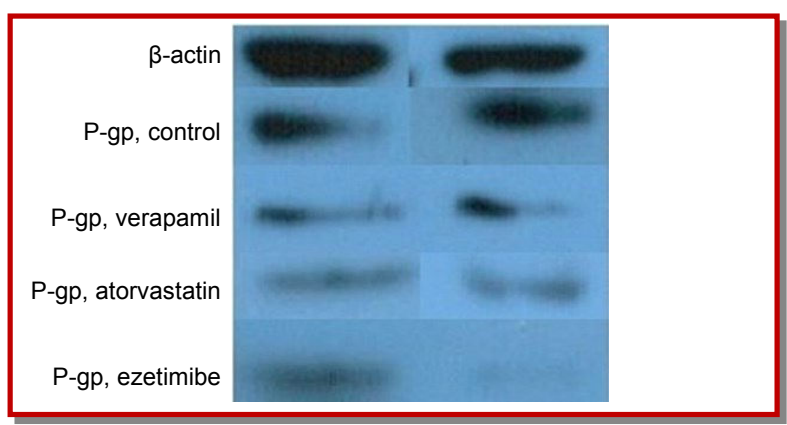

Figure 4: Western blot of P-gp and $\beta$-actin in atorvastatin, ezetimibe $(100 \mu \mathrm{M})$, and verapamil $(300 \mu \mathrm{M})$ treated and control Caco-2 cells (bands of 2 samples of each are shown)

atorvastatin inhibited digoxin secretion in Caco-2 cells (transport from the basolateral to apical aspect of the monolayer) by $58 \%$, equivalent to the inhibition observed with verapamil (Boyd et al., 2000). Another study showed that atorvastatin increased the bioavailability of digoxin (Lennernäs, 2003), which may be explained by the inhibition of P-gp by atorvastatin.

Ezetimibe-treated Caco- 2 cells exhibited more accumulation of Rho123 than cells treated with control. Rho123 is a P-gp substrate, and its accumulation may be due to the inhibitory effect of ezetimibe on P-gp. In contrast, in the rat SPIP test, 10 and $100 \mu \mathrm{M}$ ezetimibe increased the $\mathrm{P}_{\text {eff }}$ of digoxin by $91 \%$ relative to digoxin alone treated cells. Although $0.2 \mu \mathrm{M}$ ezetimibe increased the $\mathrm{P}_{\text {eff }}$ of digoxin, the effect was not statistically significant. Digoxin is a well-known substrate of P-gp and enhancement of its permeability may be due to inhibition of $\mathrm{P}$ gp efflux by ezetimibe. The inhibition by ezetimibe in situ on P-gp was not dose dependent, and the $P_{\text {eff }}$ of 


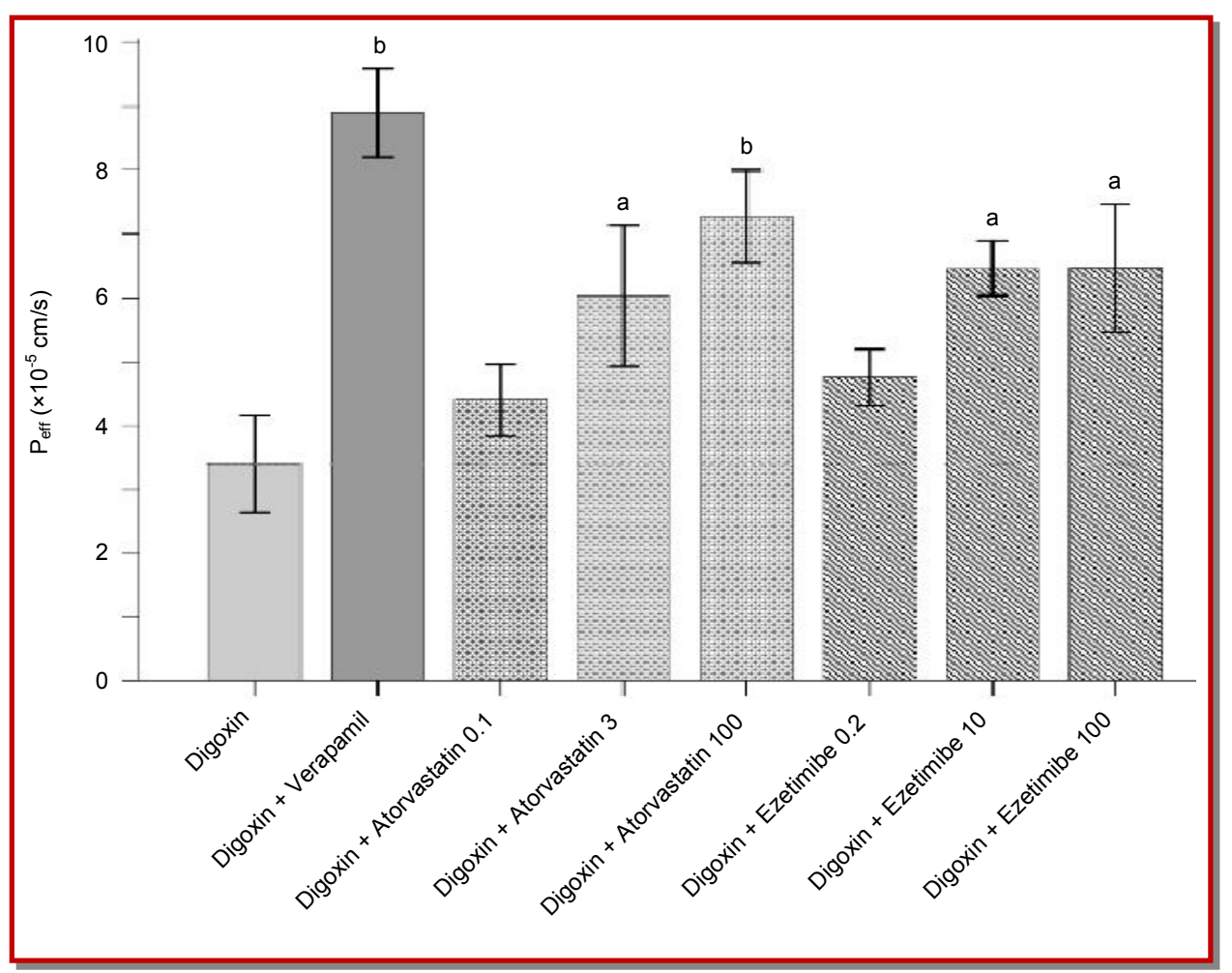

Figure 5: Effects of different concentrations of atorvastatin $(0.1,3$ and $100 \mu \mathrm{M})$, ezetimibe $(0.2,10$ and $100 \mu \mathrm{M})$ and verapamil (P-gp inhibition control) on the effective permeability $\left(\mathrm{P}_{\text {eff }}\right)$ of digoxin compared to the control group (digoxin) $(\mathrm{n}=3)$. Bars show mean \pm SD. ${ }^{a}$ and ${ }^{b}$ represent significantly different from control (digoxin) group $(\mathrm{p}<0.05$ and $\mathrm{p}<0.01$ respectively)

digoxin was approximately the same at ezetimibe concentrations greater than $10 \mu \mathrm{M}$. P-gp expression in Caco-2 cells treated with $100 \mu \mathrm{M}$ ezetimibe was less dense than that in non-treated cells, illustrating the inhibitory effects of ezetimibe on P-gp.

Ezetimibe is a biopharmaceutics classification system (BCS) class II drug with high permeability, and it undergoes rapid first-pass metabolism and P-gp efflux (Bandyopadhyay et al., 2012). To our knowledge, after oral administration of ezetimibe, it is released from the dosage form in the upper small intestine, where it is taken up into enterocytes based on the regional expression and activity of P-gp and multidrug resistant protein 2 (MRP2) (Oswald et al., 2006a). The drug is mainly excreted in the feces and is eliminated from the organism after approximately 10 days, with an elimination half-life of approximately 1 day (Bandyopadhyay et al., 2012). Oswald et al. found moderate interaction of ezetimibe with P-gp and MRP2, but the affinity of ezetimibe glucuronide for P-gp in vitro was low. This study was performed using transfected Madine Darby Canine Kidney (MDCKII), Lewis lung carcinoma (LLC), and 2008 (human ovarian carcinoma) cells. Recent studies demonstrated an important role of P-gp on the bioavailability and presystemic elimination of ezetimibe. It was shown that consistent elevation of serum ezetimibe concentrations could result from inhibition of P-gp and MRP2 by gemfibrozil, feno- fibrate and cyclosporine (Suchy et al., 2011). They also showed that up-regulation of intestinal P-gp in healthy subjects was associated with markedly decreased serum concentrations and a sterol-lowering effect of ezetimibe (Suchy et al., 2011; Bandyopadhyay et al., 2012). In another study, the intestinal expression of ATP-binding cassette, subfamily B, member 1 transporter (ABCB1) was inversely correlated with the effects of ezetimibe on plant sterol serum concentrations. The disposition and the cholesterol-lowering effect of ezetimibe were markedly influenced by the activity of intestinal efflux transport proteins, P-gp, and MDR2 (Oswald et al., 2006b). Oswald et al. concluded that the extent of oral absorption of ezetimibe is controlled by intestinal P-gp and that this efflux transport protein is involved, at least in part, in the long-lasting recirculation of the drug to the pharmacodynamic sites of action (Oswald et al., 2006a). Co-administration of ezetimibe and cyclosporine resulted in enhancement of the blood plasma levels of cyclosporine (Oswald et al., 2007). Cyclosporine is a known substrate of P-gp (Wessler et al., 2013), and the increase in its levels may be due to the Pgp inhibitory effect of ezetimibe.

The clinical dosage ranges for atorvastatin and ezetimibe are $10-80 \mathrm{mg} /$ day and up to $10 \mathrm{mg} /$ day (Lennernäs, 2003), respectively, which are within the concentration ranges used in this study. Therefore, atorvastatin and ezetimibe may inhibit P-gp at doses 
used clinically. Co-administration and interaction of atorvastatin and ezetimibe with other drugs that are Pgp substrates may result in the occurrence of adverse drug reactions owing to increases in drug absorption and the concentration in blood plasma or tissues. The DDIs become more important when the therapeutic index (the margin between effectiveness and toxicity) of the drug is narrow (e.g., digoxin).

\section{Conclusion}

Atorvastatin and ezetimibe inhibited P-gp efflux activity in vitro and in situ. The inhibitory effect of atorvastatin was dose-dependent (0.1-100 $\mu \mathrm{M})$, whereas the effect of ezetimibe $(0.2-100 \mu \mathrm{M})$ was not dosedependent. Both drugs down-regulated the expression of P-gp in vitro. The inhibitory effects of atorvastatin and ezetimibe on P-gp should be considered when predicting potential DDIs when drugs are coadministered with drugs that are P-gp substrates.

\section{Ethical Issue}

All experiments were conducted in accordance with the humanity and animal ethic protocols. The animal study protocols were registered by the research ethical committee of Tabriz University of Medical Sciences (Ref No: TBZMED.REC.1394.378).

\section{Conflict of Interest}

Authors declare no conflict of interest as documented linked in HTML format of this paper.

\section{Acknowledgement}

The authors thank the members of the Drug Applied Research Center and Immunology Research Center of Tabriz University of Medical Sciences. This work was financially supported by the Drug Applied Research Center of Tabriz University of Medical Sciences (Grant No. 91-123) and is a part of the PhD thesis of Dr. Mehran Mesgari Abbasi.

\section{References}

Adams SP, Tsang M, Wright JM. Lipid lowering efficacy of atorvastatin. Cochrane Database Syst Rev. 2012; 12: CD008226.

Ando A, Sasago S, Ohzone Y, Miyamoto Y. Drug-drug interactions of a novel K-opioid receptor agonist, nalfurafine hydrochloride, involving the P-glycoprotein. Eur J Drug Metab Pharmacokinet. 2015; 2015.

Bandyopadhyay S, Katare OP, Singh B. Colloids and surfaces B. Optimized self-nano-emulsifying systems of ezetimibe with enhanced bioavailability potential using long chain and medium chain triglycerides. Biointerfaces 2012; 100: 50-61.
Boyd RA, Stern RH, Stewart BH, Wu X, Reyner EL, Zegarac EA, Randinitis EJ, Whitfield L. Atorvastatin co-administration may increase digoxin concentrations by inhibition of intestinal P-glycoprotein-mediated secretion. J Clin Pharmacol. 2000; 40: 91-98.

Horwich TB, MacLellan WR. Atorvastatin and statins in the treatment of heart failure. Expert Opin Pharmacother. 2007; 8: 3061-68.

Lennernäs $\mathrm{H}$. Clinical pharmacokinetics of atorvastatin. Clin Pharmacokinet. 2003; 42: 1141-60.

Li Y, Huang L, Zeng X, Zhong G, Ying M, Huang M, Bi H. Down-regulation of $\mathrm{P}$-gp expression and function after mulberroside A treatment: Potential role of protein kinase $C$ and NF-kappa B. Chem Biol Int. 2014; 213: 44-50.

Lioudaki E, Ganotakis ES, Mikhailidis DP. Ezetimibe: More than a low density lipoprotein cholesterol lowering drug? An update after 4 years. Curr Vasc Pharmacol. 2011; 9: 62-86.

Mohammadzadeh R, Baradaran B, Valizadeh H, Yousefi B, Zakeri-Milani P. Reduced ABCB1 expression and activity in the presence of acrylic copolymers. Adv Pharm Bull. 2014; 4: 219-24.

$\mathrm{O}^{\prime}$ Connor M, Lee C, Ellens $\mathrm{H}$, Bentz J. A novel application of $\mathrm{t}$ statistics to objectively assess the quality of IC50 fits for Pglycoprotein and other transporters. Pharm Res Per. 2015; 3: e00078.

Oswald S, Haenisch S, Fricke C, Sudhop T, Remmler C, Giessmann T, Jedlitschky G, Adam U, Dazert E, Warzok R, Wacke W, Cascorbi I, Kroemer HK, Weitschies W, Bergmann $\mathrm{K}$, Siegmund W. Intestinal expression of P-glycoprotein (ABCB1), multidrug resistance associated protein 2 (ABCC2), and uridine diphosphate-glucuronosyltransferase $1 \mathrm{~A} 1$ predicts the disposition and modulates the effects of the cholesterol absorption inhibitor ezetimibe in humans. Clin Pharmacol Ther. 2006a; 79: 206-17.

Oswald S, Westrup S, Grube M, Kroemer HK, Weitschies W, Siegmund W. Disposition and sterol-lowering effect of ezetimibe in multidrug resistance-associated protein 2deficient rats. J Pharm Exp Ther. 2006b; 318: 1293-99.

Oswald S, Koll C, Siegmund W. Disposition of the cholesterol absorption inhibitor ezetimibe in mdrla/b (_/_) mice. J Pharm Sci. 2007; 96: 3478-84.

Patel JR, Barve KH. Intestinal permeability of lamivudine using single pass intestinal perfusion. Indian J Pharm Sci. 2012; 74: 478-81.

Phan BAP, Dayspring TD, Toth PP. Ezetimibe therapy: Mechanism of action and clinical update. Vasc Health Risk Manag. 2012; 8: 415-27.

Prasad N, Bhasker S. Characterization of intestinal transport of Vincristine in rats applying in situ single pass intestinal perfusion. Pharmacologia 2012; 3: 617-21.

Rodde MS, Divase GT, Devkar TB, Tekade AR. Solubility and bioavailability enhancement of poorly aqueous soluble atorvastatin: In vitro, ex vivo, and in vivo studies. BioMed Res Int. 2014; 2014.

Suchy D, Labuzek K, Stadnicki, Okopieñ B. Ezetimibe: A new approach in hypercholesterolemia management. Pharmacol 
Reports. 2011; 63: 1335-48.

Valizadeh H, Mehtari M, Zakeri-Milani P. Evidence for enhanced intestinal absorption of digoxin by P-glycoprotein inhibitors. Trop J Pharm Res. 2012; 11: 939-45.

Vatsa R, Varanasib KVS, Arlab R, Veeraraghavanb S, Rajakb S, Murthya AN. Effect of multidose cilostazol on pharmacokinetic and lipid profile of atorvastatin in male Wistar rats. J Pharm Pharmacol. 2012; 64: 1638-45.

Wanga XD, Menga MX, Gaoa LB, Liua T, Xub Q, Zenga S. Permeation of astilbin and taxifolin in Caco-2 cell and their effects on the P-gp. Int J Pharm. 2009; 378: 1-8.

Wessler JD, Grip LT, Mendell J, Giugliano RP. The Pglycoprotein transport system and cardiovascular drugs. J Am College Cardiol. 2013; 61: 2495-502.

Williams D, Feely J. Pharmacokinetic-pharmacodynamic drug interactions with HMG-CoA reductase inhibitors. Clin Pharmacokinet. 2002; 41: 343-70.
Wu X, Whitfield LR, Stewart BH. Atorvastatin transport in the Caco-2 cell model: Contributions of P-glycoprotein and the proton-monocarboxylic acid co-transporter. Pharm Res. 2000; 17: 209-15.

Zakeri-Milani P, Valizadeh $\mathrm{H}$, Tajerzadeh $\mathrm{H}$, Azarmi $\mathrm{Y}$, Islambolchilar $\mathrm{Z}$, Barzegar $\mathrm{S}$, et al. Predicting human intestinal permeability using single-pass intestinal perfusion in rat. J Pharm Pharm Sci. 2007; 10: 368-79.

Zastrea J, Jacksona J, Bajwaa M, Ligginsb R, Iqbala F, Burta H. Enhanced cellular accumulation of a P-glycoprotein substrate, rhodamine- 123 , by Caco- 2 cells using low molecular weight methoxypolyethylene glycol-block-polycaprolactone diblock copolymers. Eur J Pharm Biopharm. 2002; 54: 299 309.

Zrieki A, Farinotti R, Buyse M. Cyclooxygenase-2 inhibitors prevent trinitrobenzene sulfonic acid-induced P-glycolprotein up-regulation in vitro and in vivo. Eur J Pharm. 2010; 636: 189-97. 


\section{Your feedback about this paper}

1. Number of times you have read this paper 0

2. Number of times you have seen the video clip 0

3. Quality of paper Click

4. Your comments

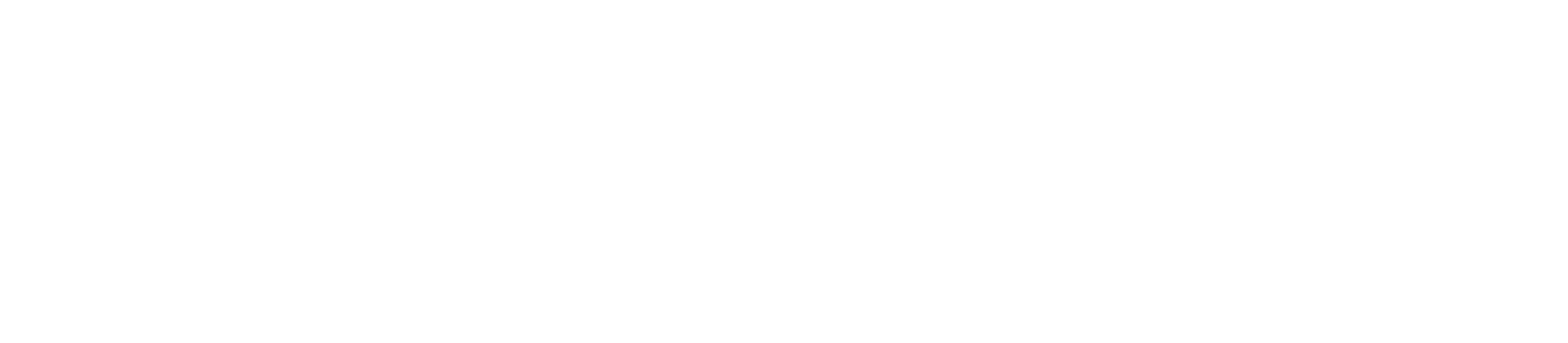

